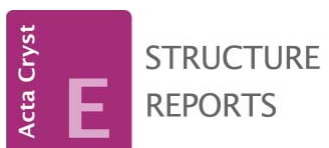

ISSN 1600-5368

\section{Crystal structure of 2-nitro-N-(5-nitro- 1,3-thiazol-2-yl)benzamide}

\author{
Rodolfo Moreno-Fuquen, ${ }^{\mathrm{a} *}$ Diego F. Sánchez $^{\mathrm{a}}$ and Javier \\ Ellena $^{b}$

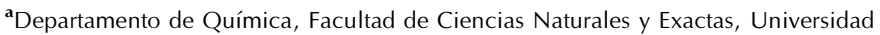 \\ del Valle, Apartado 25360, Santiago de Cali, Colombia, and ${ }^{\mathbf{b}}$ Instituto de Física de \\ São Carlos, IFSC, Universidade de São Paulo, USP, São Carlos, SP, Brazil. \\ *Correspondence e-mail: rodimo26@yahoo.es
}

Received 25 October 2014; accepted 5 November 2014

Edited by A. J. Lough, University of Toronto, Canada

In the title compound, $\mathrm{C}_{10} \mathrm{H}_{6} \mathrm{~N}_{4} \mathrm{O}_{5} \mathrm{~S}$, the mean plane of the non- $\mathrm{H}$ atoms of the central amide fragment $\mathrm{C}-\mathrm{N}-\mathrm{C}(=\mathrm{O})-$ $\mathrm{C}[$ r.m.s. deviation $=0.0294 \AA$ ] forms dihedral angles of $12.48(7)$ and $46.66(9)^{\circ}$ with the planes of the thiazole and benzene rings, respectively. In the crystal, molecules are linked by $\mathrm{N}-\mathrm{H} \cdots \mathrm{O}$ hydrogen bonds, forming chains along [001]. In addition, weak $\mathrm{C}-\mathrm{H} \cdots \mathrm{O}$ hydrogen bonds link these chains, forming a two-dimensional network, containing $R_{4}^{4}(28)$ ring motifs parallel to (100).

Keywords: crystal structure; fenilbenzamidas; 5-nitro-1,3-thiazole derivative; hydrogen bonding.

CCDC reference: 1032923

\section{Related literature}

For related structures, see: Bruno et al. (2010, 2013); Liu et al. (2013). For antiviral and antiparasitic properties of thiazolides, see: Korba et al. (2008). For hydrogen-bond details, see: Nardelli (1995).

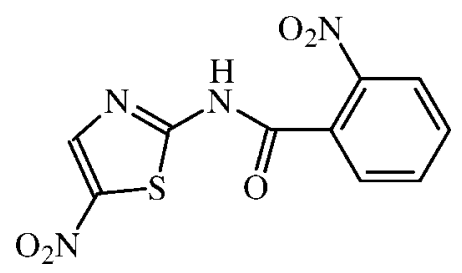

\section{Experimental}

2.1. Crystal data

$\mathrm{C}_{10} \mathrm{H}_{6} \mathrm{~N}_{4} \mathrm{O}_{5} \mathrm{~S}$

$M_{r}=294.25$

Monoclinic, $P 2_{b} / c$

$a=9.6949$ (2) A

$$
\begin{aligned}
& b=12.4192(2) \AA \\
& c=9.8763(2) \AA \\
& \beta=94.948(1)^{\circ} \\
& V=1184.70(4) \AA^{3} \\
& Z=4
\end{aligned}
$$

Mo $K \alpha$ radiation $\mu=0.30 \mathrm{~mm}^{-1}$ $T=295 \mathrm{~K}$ $0.20 \times 0.17 \times 0.12 \mathrm{~mm}$

\subsection{Data collection}

Nonius KappaCCD diffractometer 4714 measured reflections 2424 independent reflections

1867 reflections with $I>2 \sigma(I)$ $R_{\text {int }}=0.017$

\subsection{Refinement}

$R\left[F^{2}>2 \sigma\left(F^{2}\right)\right]=0.046$

$w R\left(F^{2}\right)=0.136$

$S=0.97$

2424 reflections

181 parameters

$\mathrm{H}$-atom parameters constrained

$\Delta \rho_{\max }=0.51 \mathrm{e}^{-3}$

$\Delta \rho_{\min }=-0.27$ e $\AA^{-3}$

Table 1

Hydrogen-bond geometry $\left(\AA{ }^{\circ}\right)$.

\begin{tabular}{lllll}
\hline$D-\mathrm{H} \cdots A$ & $D-\mathrm{H}$ & $\mathrm{H} \cdots A$ & $D \cdots A$ & $D-\mathrm{H} \cdots A$ \\
\hline $\mathrm{N} 3-\mathrm{H} 3 \cdots \mathrm{O} 3^{\mathrm{i}}$ & 0.86 & 2.09 & $2.949(2)$ & 175 \\
$\mathrm{C} 9-\mathrm{H} 9 \cdots 2^{\mathrm{ii}}$ & 0.93 & 2.59 & $3.193(3)$ & 123
\end{tabular}

Symmetry codes: (i) $x,-y+\frac{1}{2}, z-\frac{1}{2}$; (ii) $x, y-1, z$.

Data collection: COLLECT (Nonius, 2000); cell refinement: SCALEPACK (Otwinowski \& Minor, 1997); data reduction: DENZO (Otwinowski \& Minor, 1997) and SCALEPACK; program(s) used to solve structure: SHELXS97 (Sheldrick, 2008); program(s) used to refine structure: SHELXL97 (Sheldrick, 2008); molecular graphics: ORTEP-3 for Windows (Farrugia, 2012) and Mercury (Macrae et al., 2006); software used to prepare material for publication: Win $G X$ (Farrugia, 2012).

\section{Acknowledgements}

RMF is grateful to the Universidad del Valle, Colombia, for partial financial support.

Supporting information for this paper is available from the IUCr electronic archives (Reference: LH5737).

\section{References}

Bruno, F. P., Caira, M. R., Martin, E. C., Monti, G. A. \& Sperandeo, N. R. (2013). J. Mol. Struct. 1036, 318-325.

Bruno, F. P., Caira, M. R., Monti, G. A., Kassuha, D. E. \& Sperandeo, N. R. (2010). J. Mol. Struct. 984, 51-57.

Farrugia, L. J. (2012). J. Appl. Cryst. 45, 849-854.

Korba, B. E., Montero, A. B., Farrar, K., Gaye, K., Mukerjee, S., Ayers, M. S. \& Rossignol, J. F. (2008). Antivir. Res. 77, 56-63.

Liu, X.-W., Zhang, H., Yang, Y.-J., Li, J.-Y. \& Zhang, J.-Y. (2013). Acta Cryst. E69, o943.

Macrae, C. F., Edgington, P. R., McCabe, P., Pidcock, E., Shields, G. P., Taylor, R., Towler, M. \& van de Streek, J. (2006). J. Appl. Cryst. 39, 453-457.

Nardelli, M. (1995). J. Appl. Cryst. 28, 659.

Nonius (2000). COLLECT. Nonius BV, Delft, The Netherlands.

Otwinowski, Z. \& Minor, W. (1997). Methods in Enzymology, Vol. 276, Macromolecular Crystallography, Part A, edited by C. W. Carter Jr \& R. M. Sweet, pp. 307-326. New York: Academic Press.

Sheldrick, G. M. (2008). Acta Cryst. A64, 112-122. 


\section{supporting information}

Acta Cryst. (2014). E70, o1252 [doi:10.1107/S1600536814024374]

\section{Crystal structure of 2-nitro-N-(5-nitro-1,3-thiazol-2-yl)benzamide Rodolfo Moreno-Fuquen, Diego F. Sánchez and Javier Ellena}

\section{S1. Comment}

The crystal structure determination of the title compound (I), is part of a study of a series of fenilbenzamidas derived from 5-nitro-1,3-thiazole carried out by our research group. Crystal structures of compounds similar to (I), such as 2-hydroxy-N-(5-nitro-2-thiazolyl)benzamide, (TIZ) (Bruno et al., 2013), 2-acetyloxy-N-(5-nitro-2-thiazolyl)benzamide, (NTZ) (Bruno et al., 2010) and N-(5-nitro-1,3-thiazol-2-yl)-4(trifluoromethyl)benzamide (NTF) (Liu et al., 2013) can be compared with (I). Both the structures of (TIZ) and (NTZ) have been reported in standard antiviral essays as potent inhibitors for hepatitis B and hepatitis $\mathrm{C}$ replication process (Korba et al., 2008). These thiazolides have also been reported as a potent antiparasitic and antiviral agents against intestinal infections caused by various parasitic protozoa (Bruno et al., 2013).

The molecular structure of (I) is shown in Fig. 1. The central amide group, $\mathrm{C} 3-\mathrm{N} 3-\mathrm{C} 4(=\mathrm{O} 3)-\mathrm{C} 5$, is essentially planar (r.m.s. deviation for all non-H atoms $=0.0294 \AA$ ) and forms dihedral angles of $12.48(7)^{\circ}$ and $46.66(9)^{\circ}$ with the thiazole ring and benzene ring, respectively. The bond lengths and the degree of planarity in the central amide group in (I) are similar to those shown in NTZ, TIZ and NTF. The nitro O1/N1/O2 and O4/N4/O5 groups form dihedral angles of $4.07(11)^{\circ}$ and $47.09(11)^{\circ}$ with the attached thiazole and benzene rings, respectively. In the crystal (Fig. 2), molecules are

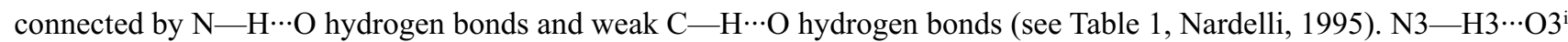
hydrogen bonds are responsible for hydrogen-bonded chains in the c-axis direction. The N3-H3 group of the amide moiety in the molecule at $(\mathrm{x}, \mathrm{y}, \mathrm{z})$ acts as a hydrogen-bond donor to $\mathrm{O} 3$ atom of the carbonyl group in the molecule at (x, $-\mathrm{y}+1 / 2, \mathrm{z}-1 / 2)$. In addition, weak hydrogen bonds $\mathrm{C} 9-\mathrm{H} 9 \cdots \mathrm{O} 2^{\mathrm{ii}}$, form chains in the b-axis direction. The $\mathrm{C} 9-\mathrm{H} 9$ group of the benzene ring in the molecule at $(\mathrm{x}, \mathrm{y}, \mathrm{z})$ acts as a hydrogen bond donor to atom $\mathrm{O} 2$ in the molecule at $(\mathrm{x}, \mathrm{y}-1, \mathrm{z})$. It is possible that for this weak hydrogen bond to occur, a rotation of the benzene ring with respect to plane formed by the central amide moiety is required. The combination of the above hydrogen bond interactions generate edge-fused $\mathrm{R}_{4}^{4}(28)$ rings.

\section{S2. Experimental}

The reagents and solvents for the synthesis were obtained from the Aldrich Chemical Co., and were used without additional purification. The title molecule was synthesized using equimolar amounts of 2-nitrobenzoyl chloride $(0.171 \mathrm{~g}$, $0.923 \mathrm{mmol})$ and 5 -nitro-1,3-thiazole $(0.134 \mathrm{~g})$. The reagents were dissolved in $7 \mathrm{~mL}$ of acetonitrile and the solution was refluxed with constant stirring for 4 hours. A after evaporation of the solvent a brown solid was obtained. The solid was washed with distilled water to remove impurities. Pale-brown crystals of good quality [m.p. 515 (1)K] suitable for singlecrystal X-ray diffraction were grown from a solution of the title compound in acetonitrile. 


\section{S3. Refinement}

All $\mathrm{H}$ atoms were positioned in geometrically idealized positions, with $\mathrm{C}-\mathrm{H}=0.93 \AA$ and $\mathrm{N}-\mathrm{H}=0.86 \AA$, and were refined using a riding-model approximation, with $U_{\text {iso }}(\mathrm{H})$ constrained to 1.2 times $U_{\mathrm{eq}}$ of the respective parent atom.

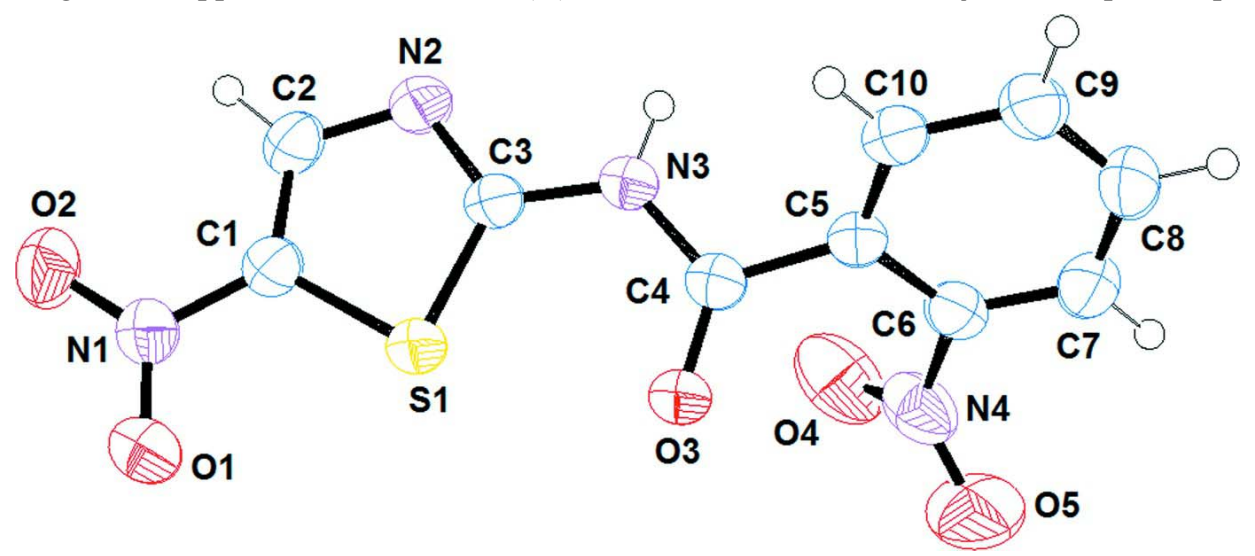

\section{Figure 1}

The molecular structure of (I) with displacement ellipsoids drawn at the $50 \%$ probability level. $\mathrm{H}$ atoms are shown as spheres of arbitrary radius.

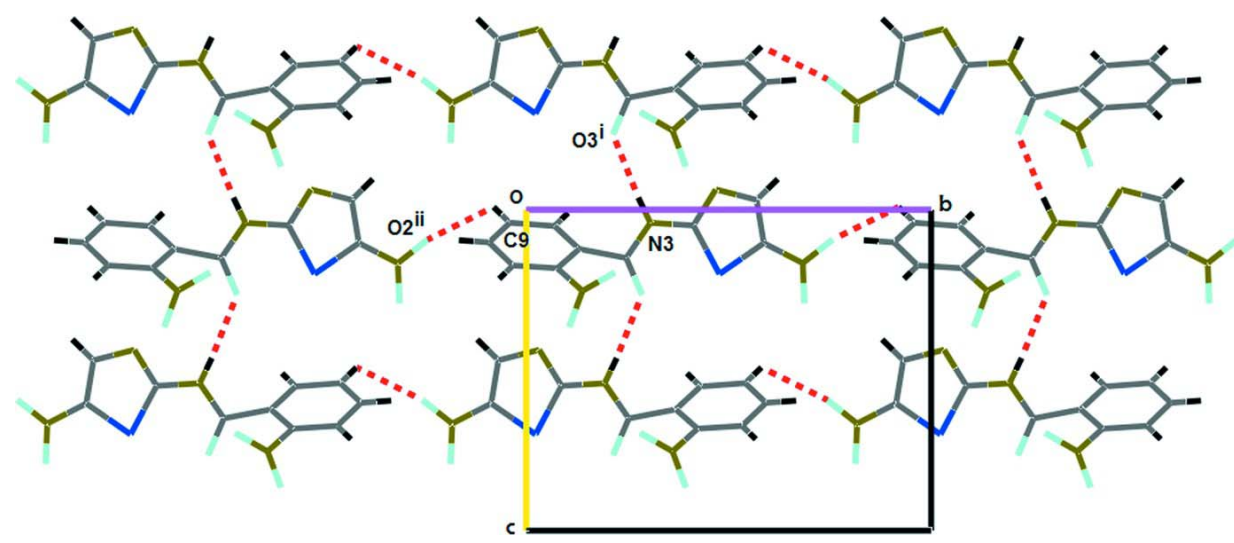

\section{Figure 2}

Part of the crystal structure of (I), showing the formation of $\mathrm{R}_{4}^{4}(28)$ rings within a 2-D hydrogen-bonded network (dashed lines) running parallel to (100) [Symmetry codes: (i) $x,-y+1 / 2, z-1 / 2$; (ii) $x, y-1, z]$.

\section{2-Nitro-N-(5-nitro-1,3-thiazol-2-yl)benzamide}

\section{Crystal data}

$\mathrm{C}_{10} \mathrm{H}_{6} \mathrm{~N}_{4} \mathrm{O}_{5} \mathrm{~S}$

$M_{r}=294.25$

Monoclinic, $P 2_{1} / c$

Hall symbol: -P $2 \mathrm{ybc}$

$a=9.6949$ (2) $\AA$

$b=12.4192(2) \AA$

$c=9.8763(2) \AA$

$\beta=94.948(1)^{\circ}$

$V=1184.70(4) \AA^{3}$

$Z=4$
$F(000)=600$

$D_{\mathrm{x}}=1.650 \mathrm{Mg} \mathrm{m}^{-3}$

Melting point: $515(1) \mathrm{K}$

Mo $K \alpha$ radiation, $\lambda=0.71073 \AA$

Cell parameters from 2543 reflections

$\theta=2.9-26.4^{\circ}$

$\mu=0.30 \mathrm{~mm}^{-1}$

$T=295 \mathrm{~K}$

Block, brown

$0.20 \times 0.17 \times 0.12 \mathrm{~mm}$ 


\section{Data collection}

Nonius KappaCCD diffractometer

Radiation source: fine-focus sealed tube Graphite monochromator

CCD rotation images, thick slices scans

4714 measured reflections

2424 independent reflections

\section{Refinement}

Refinement on $F^{2}$

Least-squares matrix: full

$R\left[F^{2}>2 \sigma\left(F^{2}\right)\right]=0.046$

$w R\left(F^{2}\right)=0.136$

$S=0.97$

2424 reflections

181 parameters

0 restraints

Primary atom site location: structure-invariant direct methods
1867 reflections with $I>2 \sigma(I)$

$R_{\text {int }}=0.017$

$\theta_{\max }=26.4^{\circ}, \theta_{\min }=3.3^{\circ}$

$h=-12 \rightarrow 12$

$k=-15 \rightarrow 15$

$l=-12 \rightarrow 12$

\section{Special details}

Geometry. All esds (except the esd in the dihedral angle between two l.s. planes) are estimated using the full covariance matrix. The cell esds are taken into account individually in the estimation of esds in distances, angles and torsion angles; correlations between esds in cell parameters are only used when they are defined by crystal symmetry. An approximate (isotropic) treatment of cell esds is used for estimating esds involving l.s. planes.

Refinement. Refinement of $F^{2}$ against ALL reflections. The weighted $R$-factor $w R$ and goodness of fit $S$ are based on $F^{2}$, conventional $R$-factors $R$ are based on $F$, with $F$ set to zero for negative $F^{2}$. The threshold expression of $F^{2}>\sigma\left(F^{2}\right)$ is used only for calculating $R$-factors $(\mathrm{gt})$ etc. and is not relevant to the choice of reflections for refinement. $R$-factors based on $F^{2}$ are statistically about twice as large as those based on $F$, and $R$-factors based on ALL data will be even larger.

Fractional atomic coordinates and isotropic or equivalent isotropic displacement parameters $\left(\AA^{2}\right)$

\begin{tabular}{lllll}
\hline & $x$ & $y$ & $z$ & $U_{\text {iso }} * / U_{\text {eq }}$ \\
\hline S1 & $0.33684(6)$ & $0.47701(4)$ & $0.20035(5)$ & $0.0441(2)$ \\
O3 & $0.22493(17)$ & $0.28583(12)$ & $0.26475(14)$ & $0.0502(4)$ \\
$\mathrm{N} 1$ & $0.4288(2)$ & $0.68288(15)$ & $0.16636(19)$ & $0.0473(4)$ \\
$\mathrm{N} 2$ & $0.3021(2)$ & $0.46806(14)$ & $-0.06343(17)$ & $0.0463(5)$ \\
$\mathrm{N} 3$ & $0.24829(18)$ & $0.30650(13)$ & $0.04092(17)$ & $0.0420(4)$ \\
$\mathrm{H} 3$ & 0.2390 & 0.2766 & -0.0379 & $0.050^{*}$ \\
O2 & $0.4626(2)$ & $0.75643(14)$ & $0.09317(19)$ & $0.0648(5)$ \\
C6 & $0.0858(2)$ & $0.07681(18)$ & $0.1823(2)$ & $0.0449(5)$ \\
C5 & $0.1907(2)$ & $0.13054(16)$ & $0.12231(19)$ & $0.0400(5)$ \\
C10 & $0.2795(2)$ & $0.06972(18)$ & $0.0508(2)$ & $0.0470(5)$ \\
H10 & 0.3508 & 0.1032 & 0.0096 & $0.056^{*}$ \\
C1 & $0.3749(2)$ & $0.58660(17)$ & $0.1039(2)$ & $0.0421(5)$ \\
C3 & $0.2907(2)$ & $0.41287(16)$ & $0.04889(19)$ & $0.0395(5)$ \\
C4 & $0.2206(2)$ & $0.24675(16)$ & $0.1504(2)$ & $0.0401(5)$ \\
O1 & $0.4402(2)$ & $0.68685(15)$ & $0.29129(17)$ & $0.0706(5)$ \\
C8 & $0.1593(3)$ & $-0.09204(19)$ & $0.1018(2)$ & $0.0576(6)$ \\
H8 & 0.1498 & -0.1664 & 0.0950 & $0.069 *$
\end{tabular}




$\begin{array}{lllll}\mathrm{N} 4 & -0.0150(2) & 0.1388(2) & 0.2531(3) & 0.0650(6) \\ \mathrm{C} 2 & 0.3492(2) & 0.56887(17) & -0.0307(2) & 0.0458(5) \\ \mathrm{H} 2 & 0.3624 & 0.6212 & -0.0957 & 0.055^{*} \\ \mathrm{C} 9 & 0.2627(3) & -0.04074(18) & 0.0405(2) & 0.0536(6) \\ \mathrm{H} 9 & 0.3224 & -0.0807 & -0.0086 & 0.064^{*} \\ \text { O5 } & -0.0429(3) & 0.1061(2) & 0.3650(3) & 0.1011(8) \\ \text { C7 } & 0.0691(3) & -0.03301(19) & 0.1736(2) & 0.0545(6) \\ \text { H7 } & -0.0015 & -0.0670 & 0.2153 & 0.065^{*} \\ \text { O4 } & -0.0647(2) & 0.2185(2) & 0.1957(3) & 0.0905(7)\end{array}$

Atomic displacement parameters $\left(\AA^{2}\right)$

\begin{tabular}{lllllll}
\hline & $U^{11}$ & $U^{22}$ & $U^{33}$ & $U^{12}$ & $U^{13}$ & $U^{23}$ \\
\hline S1 & $0.0603(4)$ & $0.0397(3)$ & $0.0326(3)$ & $-0.0048(2)$ & $0.0058(2)$ & $-0.0005(2)$ \\
O3 & $0.0742(11)$ & $0.0441(8)$ & $0.0327(8)$ & $-0.0080(7)$ & $0.0074(7)$ & $-0.0032(6)$ \\
N1 & $0.0534(11)$ & $0.0408(10)$ & $0.0477(11)$ & $-0.0016(8)$ & $0.0051(8)$ & $0.0001(8)$ \\
N2 & $0.0615(11)$ & $0.0440(10)$ & $0.0331(9)$ & $-0.0017(8)$ & $0.0025(8)$ & $0.0043(7)$ \\
N3 & $0.0559(11)$ & $0.0386(9)$ & $0.0318(9)$ & $-0.0032(8)$ & $0.0054(7)$ & $-0.0030(7)$ \\
O2 & $0.0838(13)$ & $0.0436(9)$ & $0.0664(11)$ & $-0.0114(8)$ & $0.0033(9)$ & $0.0092(8)$ \\
C6 & $0.0490(12)$ & $0.0471(12)$ & $0.0388(11)$ & $-0.0053(10)$ & $0.0044(9)$ & $-0.0067(9)$ \\
C5 & $0.0490(12)$ & $0.0394(11)$ & $0.0313(10)$ & $-0.0029(9)$ & $0.0019(8)$ & $-0.0002(8)$ \\
C10 & $0.0580(13)$ & $0.0458(12)$ & $0.0382(11)$ & $0.0012(10)$ & $0.0100(10)$ & $0.0018(9)$ \\
C1 & $0.0463(12)$ & $0.0400(11)$ & $0.0403(11)$ & $-0.0003(9)$ & $0.0060(9)$ & $0.0015(8)$ \\
C3 & $0.0453(11)$ & $0.0400(11)$ & $0.0337(10)$ & $0.0002(9)$ & $0.0061(8)$ & $0.0009(8)$ \\
C4 & $0.0447(11)$ & $0.0405(11)$ & $0.0349(10)$ & $-0.0008(9)$ & $0.0029(8)$ & $-0.0020(8)$ \\
O1 & $0.1054(15)$ & $0.0597(11)$ & $0.0466(10)$ & $-0.0183(10)$ & $0.0060(9)$ & $-0.0093(8)$ \\
C8 & $0.0830(18)$ & $0.0375(12)$ & $0.0515(14)$ & $-0.0025(12)$ & $0.0019(13)$ & $0.0042(10)$ \\
N4 & $0.0504(12)$ & $0.0725(16)$ & $0.0738(15)$ & $-0.0156(11)$ & $0.0157(11)$ & $-0.0284(12)$ \\
C2 & $0.0539(13)$ & $0.0413(12)$ & $0.0426(12)$ & $-0.0009(10)$ & $0.0074(10)$ & $0.0081(9)$ \\
C9 & $0.0742(16)$ & $0.0411(12)$ & $0.0464(13)$ & $0.0118(11)$ & $0.0107(11)$ & $0.0009(10)$ \\
O5 & $0.0955(17)$ & $0.130(2)$ & $0.0857(16)$ & $-0.0291(15)$ & $0.0539(14)$ & $-0.0220(14)$ \\
C7 & $0.0674(15)$ & $0.0508(14)$ & $0.0458(13)$ & $-0.0162(11)$ & $0.0072(11)$ & $0.0017(10)$ \\
O4 & $0.0802(15)$ & $0.0786(15)$ & $0.1127(18)$ & $0.0220(12)$ & $0.0079(13)$ & $-0.0326(14)$ \\
& & & & & & \\
\hline
\end{tabular}

Geometric parameters $\left(\AA,{ }^{\circ}\right)$

\begin{tabular}{llll}
\hline $\mathrm{S} 1-\mathrm{C} 3$ & $1.720(2)$ & $\mathrm{C} 5-\mathrm{C} 10$ & $1.384(3)$ \\
$\mathrm{S} 1-\mathrm{C} 1$ & $1.720(2)$ & $\mathrm{C} 5-\mathrm{C} 4$ & $1.493(3)$ \\
$\mathrm{O} 3-\mathrm{C} 4$ & $1.227(2)$ & $\mathrm{C} 10-\mathrm{C} 9$ & $1.384(3)$ \\
$\mathrm{N} 1-\mathrm{O} 2$ & $1.227(2)$ & $\mathrm{C} 10-\mathrm{H} 10$ & 0.9300 \\
$\mathrm{~N} 1-\mathrm{O} 1$ & $1.230(2)$ & $\mathrm{C} 1-\mathrm{C} 2$ & $1.349(3)$ \\
$\mathrm{N} 1-\mathrm{C} 1$ & $1.424(3)$ & $\mathrm{C} 8-\mathrm{C} 9$ & $1.372(4)$ \\
$\mathrm{N} 2-\mathrm{C} 3$ & $1.317(2)$ & $\mathrm{C} 8-\mathrm{C} 7$ & $1.383(4)$ \\
$\mathrm{N} 2-\mathrm{C} 2$ & $1.362(3)$ & $\mathrm{C} 8-\mathrm{H} 8$ & 0.9300 \\
$\mathrm{~N} 3-\mathrm{C} 4$ & $1.357(3)$ & $\mathrm{N} 4-\mathrm{O} 4$ & $1.218(4)$ \\
$\mathrm{N} 3-\mathrm{C} 3$ & $1.384(3)$ & $\mathrm{N} 4-\mathrm{O} 5$ & $1.230(3)$ \\
$\mathrm{N} 3-\mathrm{H} 3$ & 0.8600 & $\mathrm{C} 2-\mathrm{H} 2$ & 0.9300 \\
$\mathrm{C} 6-\mathrm{C} 7$ & $1.375(3)$ & $\mathrm{C}-\mathrm{H} 9$ & 0.9300
\end{tabular}




\begin{tabular}{|c|c|c|c|}
\hline $\mathrm{C} 6-\mathrm{C} 5$ & $1.391(3)$ & $\mathrm{C} 7-\mathrm{H} 7$ & 0.9300 \\
\hline $\mathrm{C} 6-\mathrm{N} 4$ & $1.468(3)$ & & \\
\hline $\mathrm{C} 3-\mathrm{S} 1-\mathrm{C} 1$ & $86.39(10)$ & $\mathrm{N} 2-\mathrm{C} 3-\mathrm{S} 1$ & $117.22(16)$ \\
\hline $\mathrm{O} 2-\mathrm{N} 1-\mathrm{O} 1$ & $123.74(19)$ & $\mathrm{N} 3-\mathrm{C} 3-\mathrm{S} 1$ & $123.06(15)$ \\
\hline $\mathrm{O} 2-\mathrm{N} 1-\mathrm{C} 1$ & $118.47(18)$ & $\mathrm{O} 3-\mathrm{C} 4-\mathrm{N} 3$ & $121.59(19)$ \\
\hline $\mathrm{O} 1-\mathrm{N} 1-\mathrm{C} 1$ & $117.78(18)$ & $\mathrm{O} 3-\mathrm{C} 4-\mathrm{C} 5$ & $122.95(18)$ \\
\hline $\mathrm{C} 3-\mathrm{N} 2-\mathrm{C} 2$ & $109.22(17)$ & $\mathrm{N} 3-\mathrm{C} 4-\mathrm{C} 5$ & $115.42(17)$ \\
\hline $\mathrm{C} 4-\mathrm{N} 3-\mathrm{C} 3$ & $123.71(17)$ & $\mathrm{C} 9-\mathrm{C} 8-\mathrm{C} 7$ & $119.9(2)$ \\
\hline $\mathrm{C} 4-\mathrm{N} 3-\mathrm{H} 3$ & 118.1 & $\mathrm{C} 9-\mathrm{C} 8-\mathrm{H} 8$ & 120.0 \\
\hline $\mathrm{C} 3-\mathrm{N} 3-\mathrm{H} 3$ & 118.1 & $\mathrm{C} 7-\mathrm{C} 8-\mathrm{H} 8$ & 120.0 \\
\hline $\mathrm{C} 7-\mathrm{C} 6-\mathrm{C} 5$ & $122.4(2)$ & $\mathrm{O} 4-\mathrm{N} 4-\mathrm{O} 5$ & $125.2(3)$ \\
\hline $\mathrm{C} 7-\mathrm{C} 6-\mathrm{N} 4$ & $118.1(2)$ & $\mathrm{O} 4-\mathrm{N} 4-\mathrm{C} 6$ & $117.3(2)$ \\
\hline $\mathrm{C} 5-\mathrm{C} 6-\mathrm{N} 4$ & $119.5(2)$ & $\mathrm{O} 5-\mathrm{N} 4-\mathrm{C} 6$ & $117.5(3)$ \\
\hline $\mathrm{C} 10-\mathrm{C} 5-\mathrm{C} 6$ & $117.7(2)$ & $\mathrm{C} 1-\mathrm{C} 2-\mathrm{N} 2$ & $114.44(18)$ \\
\hline $\mathrm{C} 10-\mathrm{C} 5-\mathrm{C} 4$ & $120.18(19)$ & $\mathrm{C} 1-\mathrm{C} 2-\mathrm{H} 2$ & 122.8 \\
\hline $\mathrm{C} 6-\mathrm{C} 5-\mathrm{C} 4$ & $121.46(18)$ & $\mathrm{N} 2-\mathrm{C} 2-\mathrm{H} 2$ & 122.8 \\
\hline $\mathrm{C} 5-\mathrm{C} 10-\mathrm{C} 9$ & $120.3(2)$ & $\mathrm{C} 8-\mathrm{C} 9-\mathrm{C} 10$ & $120.9(2)$ \\
\hline $\mathrm{C} 5-\mathrm{C} 10-\mathrm{H} 10$ & 119.9 & $\mathrm{C} 8-\mathrm{C} 9-\mathrm{H} 9$ & 119.6 \\
\hline $\mathrm{C} 9-\mathrm{C} 10-\mathrm{H} 10$ & 119.9 & $\mathrm{C} 10-\mathrm{C} 9-\mathrm{H} 9$ & 119.6 \\
\hline $\mathrm{C} 2-\mathrm{C} 1-\mathrm{N} 1$ & $126.41(19)$ & $\mathrm{C} 6-\mathrm{C} 7-\mathrm{C} 8$ & $118.8(2)$ \\
\hline $\mathrm{C} 2-\mathrm{C} 1-\mathrm{S} 1$ & $112.72(16)$ & $\mathrm{C} 6-\mathrm{C} 7-\mathrm{H} 7$ & 120.6 \\
\hline $\mathrm{N} 1-\mathrm{C} 1-\mathrm{S} 1$ & $120.87(16)$ & $\mathrm{C} 8-\mathrm{C} 7-\mathrm{H} 7$ & 120.6 \\
\hline $\mathrm{N} 2-\mathrm{C} 3-\mathrm{N} 3$ & $119.67(18)$ & & \\
\hline $\mathrm{C} 7-\mathrm{C} 6-\mathrm{C} 5-\mathrm{C} 10$ & $0.5(3)$ & $\mathrm{C} 3-\mathrm{N} 3-\mathrm{C} 4-\mathrm{O} 3$ & $3.8(3)$ \\
\hline $\mathrm{N} 4-\mathrm{C} 6-\mathrm{C} 5-\mathrm{C} 10$ & $-176.9(2)$ & $\mathrm{C} 3-\mathrm{N} 3-\mathrm{C} 4-\mathrm{C} 5$ & $-173.84(18)$ \\
\hline $\mathrm{C} 7-\mathrm{C} 6-\mathrm{C} 5-\mathrm{C} 4$ & $-170.5(2)$ & $\mathrm{C} 10-\mathrm{C} 5-\mathrm{C} 4-\mathrm{O} 3$ & $-127.2(2)$ \\
\hline $\mathrm{N} 4-\mathrm{C} 6-\mathrm{C} 5-\mathrm{C} 4$ & $12.1(3)$ & $\mathrm{C} 6-\mathrm{C} 5-\mathrm{C} 4-\mathrm{O} 3$ & $43.6(3)$ \\
\hline $\mathrm{C} 6-\mathrm{C} 5-\mathrm{C} 10-\mathrm{C} 9$ & $0.1(3)$ & $\mathrm{C} 10-\mathrm{C} 5-\mathrm{C} 4-\mathrm{N} 3$ & $50.3(3)$ \\
\hline $\mathrm{C} 4-\mathrm{C} 5-\mathrm{C} 10-\mathrm{C} 9$ & $171.2(2)$ & $\mathrm{C} 6-\mathrm{C} 5-\mathrm{C} 4-\mathrm{N} 3$ & $-138.8(2)$ \\
\hline $\mathrm{O} 2-\mathrm{N} 1-\mathrm{C} 1-\mathrm{C} 2$ & $-4.0(3)$ & $\mathrm{C} 7-\mathrm{C} 6-\mathrm{N} 4-\mathrm{O} 4$ & $-131.6(3)$ \\
\hline $\mathrm{O} 1-\mathrm{N} 1-\mathrm{C} 1-\mathrm{C} 2$ & $176.9(2)$ & $\mathrm{C} 5-\mathrm{C} 6-\mathrm{N} 4-\mathrm{O} 4$ & $45.9(3)$ \\
\hline $\mathrm{O} 2-\mathrm{N} 1-\mathrm{C} 1-\mathrm{S} 1$ & $175.42(16)$ & $\mathrm{C} 7-\mathrm{C} 6-\mathrm{N} 4-\mathrm{O} 5$ & $48.1(3)$ \\
\hline $\mathrm{O} 1-\mathrm{N} 1-\mathrm{C} 1-\mathrm{S} 1$ & $-3.7(3)$ & $\mathrm{C} 5-\mathrm{C} 6-\mathrm{N} 4-\mathrm{O} 5$ & $-134.4(2)$ \\
\hline $\mathrm{C} 3-\mathrm{S} 1-\mathrm{C} 1-\mathrm{C} 2$ & $1.01(17)$ & $\mathrm{N} 1-\mathrm{C} 1-\mathrm{C} 2-\mathrm{N} 2$ & $177.9(2)$ \\
\hline $\mathrm{C} 3-\mathrm{S} 1-\mathrm{C} 1-\mathrm{N} 1$ & $-178.52(19)$ & $\mathrm{S} 1-\mathrm{C} 1-\mathrm{C} 2-\mathrm{N} 2$ & $-1.6(3)$ \\
\hline $\mathrm{C} 2-\mathrm{N} 2-\mathrm{C} 3-\mathrm{N} 3$ & $-178.09(18)$ & $\mathrm{C} 3-\mathrm{N} 2-\mathrm{C} 2-\mathrm{C} 1$ & $1.4(3)$ \\
\hline $\mathrm{C} 2-\mathrm{N} 2-\mathrm{C} 3-\mathrm{S} 1$ & $-0.6(3)$ & $\mathrm{C} 7-\mathrm{C} 8-\mathrm{C} 9-\mathrm{C} 10$ & $0.8(4)$ \\
\hline $\mathrm{C} 4-\mathrm{N} 3-\mathrm{C} 3-\mathrm{N} 2$ & $-173.3(2)$ & $\mathrm{C} 5-\mathrm{C} 10-\mathrm{C} 9-\mathrm{C} 8$ & $-0.7(3)$ \\
\hline $\mathrm{C} 4-\mathrm{N} 3-\mathrm{C} 3-\mathrm{S} 1$ & $9.4(3)$ & $\mathrm{C} 5-\mathrm{C} 6-\mathrm{C} 7-\mathrm{C} 8$ & $-0.5(3)$ \\
\hline $\mathrm{C} 1-\mathrm{S} 1-\mathrm{C} 3-\mathrm{N} 2$ & $-0.22(18)$ & $\mathrm{N} 4-\mathrm{C} 6-\mathrm{C} 7-\mathrm{C} 8$ & $177.0(2)$ \\
\hline $\mathrm{C} 1-\mathrm{S} 1-\mathrm{C} 3-\mathrm{N} 3$ & $177.18(19)$ & $\mathrm{C} 9-\mathrm{C} 8-\mathrm{C} 7-\mathrm{C} 6$ & $-0.2(4)$ \\
\hline
\end{tabular}

Hydrogen-bond geometry $\left(A,{ }^{\circ}\right)$

\begin{tabular}{lllll}
\hline$D-\mathrm{H} \cdots A$ & $D-\mathrm{H}$ & $\mathrm{H} \cdots A$ & $D \cdots A$ & $D-\mathrm{H} \cdots A$ \\
\hline $\mathrm{N} 3-\mathrm{H} 3 \cdots \mathrm{O}^{\mathrm{i}}$ & 0.86 & 2.09 & $2.949(2)$ & 175
\end{tabular}


$\mathrm{C} 9-\mathrm{H} 9 \cdots \mathrm{O} 2^{\mathrm{ii}}$

0.93

2.59

$3.193(3)$

123

Symmetry codes: (i) $x,-y+1 / 2, z-1 / 2$; (ii) $x, y-1, z$. 